\title{
ARTICLE The role of orphan receptor GPR139 in neuropsychiatric behavior
}

\author{
Maria Dao ${ }^{1}$, Hannah M. Stoveken ${ }^{1}$, Yan Cao $^{1}$ and Kirill A. Martemyanov (D) ${ }^{1}$
}

\begin{abstract}
Orphan G protein Coupled Receptors (GPCRs) present attractive targets both for understanding neuropsychiatric diseases and for development of novel therapeutics. GPR139 is an orphan GPCR expressed in select brain circuits involved in controlling movement, motivation and reward. It has been linked to the opioid and dopamine neuromodulatory systems; however, its role in animal behavior and neuropsychiatric processes is poorly understood. Here we present a comprehensive behavioral characterization of a mouse model with a GPR139 null mutation. We show that loss of GPR139 in mice results in delayed onset hyperactivity and prominent neuropsychiatric manifestations including elevated stereotypy, increased anxiety-related traits, delayed acquisition of operant responsiveness, disruption of cued fear conditioning and social interaction deficits. Furthermore, mice lacking GPR139 exhibited complete loss of pre-pulse inhibition and developed spontaneous 'hallucinogenic' head-twitches, altogether suggesting schizophrenia-like symptomatology. Remarkably, a number of these behavioral deficits could be rescued by the administration of $\mu-$ opioid and D2 dopamine receptor (D2R) antagonists: naltrexone and haloperidol, respectively, suggesting that loss of neuropsychiatric manifestations in mice lacking GPR139 are driven by opioidergic and dopaminergic hyper-functionality. The inhibitory influence of GPR139 on D2R signaling was confirmed in cell-based functional assays. These observations define the role of GPR139 in controlling behavior and implicate in vivo actions of this receptor in the neuropsychiatric process with schizophrenialike pathology.
\end{abstract}

Neuropsychopharmacology (2022) 47:902-913; https://doi.org/10.1038/s41386-021-00962-2

\section{INTRODUCTION}

Neuromodulation endows brain circuits with the capacity for plasticity, adjusting neural responses based on changing circumstance [1]. Mammalian nervous systems feature dozens of neuromodulatory systems with distinct properties, physiological effects and selective distribution across neural circuits. Collectively, they modulate virtually every aspect of behavior including cognition, emotional states, social interactions and feeding [2,3]. Dysfunction in the processing of neuromodulatory signals is strongly associated with a variety of neuropsychiatric conditions [3].

The effects of most, if not all, neuromodulators are mediated by G protein coupled Receptors (GPCRs), which comprise the most extensive family of surface receptors in mammals. GPCRs transduce signals via heterotrimeric $G$ proteins and $\beta$-arrestin that directly or indirectly modulate second messenger pathways and ion channels $[4,5]$. Ultimately, this signaling prominently affects neuronal excitability, firing and synaptic communication, and thereby provides the basis for neuromodulatory influence. GPCRs are also the most successful drug targets. A large fraction of currently approved medications for a variety of neuropsychiatric conditions work by activating or inhibiting GPCR signaling $[6,7]$. However, much remains to be learned about the actions of individual GPCRs in select brain circuits, in controlling behavior and in their role in neuropsychiatric disease processes [8,9]. There is a particular paucity of knowledge regarding the influence of neuromodulatory GPCR systems on cognition and sensorimotor integration and their contribution to disorders like schizophrenia, hyperactivity and attention deficit disorder.

Orphan GPCRs offer great promises for discovering new neuromodulatory systems and obtaining significant insights into the etiology of neuropsychiatric conditions [10]. They contain a group of $\sim 100$ receptors with unknown matching to neuromodulators, obscure signaling mechanisms and poorly understood physiological roles $[6,11]$. Yet, several "de-orphanization" success stories have highlighted the tremendous potential for these systems to serve as novel targets for drug discovery.

One such intriguing orphan receptor system is represented by GPR139 [12]. This receptor exhibits the hallmark features of canonical peptide receptors belonging to class A GPCRs [13, 14]. Although endogenous, physiologically relevant ligands for GPR139 have not been firmly established, it was shown to be weakly activated by aromatic amino acids and peptides derived from aMSH [15, 16]. Additionally, a number of potent and selective surrogate synthetic ligands have also been developed [17-23]. Activated GPR139 was shown to trigger $\mathrm{Ca}^{2+}$ mobilization, ERK phosphorylation and CAMP modulation [15-20, 24]. In reconstituted cells, GPR139 is capable of activating several G proteins, most notably Gaq/11, which is utilized for the transmission of physiologically relevant signals in the endogenous setting [25]. Studies have shown that GPR139 displays a selective expression pattern in the brain with prominent enrichment in neuronal circuits underlying motivated behaviors, movement control, nociception and cognition in the brain regions such as habenula,

${ }^{1}$ Department of Neuroscience, The Scripps Research Institute, 130 Scripps Way, Jupiter, FL 33458, USA

Correspondence: Kirill A. Martemyanov (kirill@scripps.edu)

Received: 4 September 2020 Revised: 1 January 2021 Accepted: 5 January 2021

Published online: 21 January 2021 
striatum, hippocampus, locus coeruleus, ventral tegmental area and dorsal root ganglia [26-28]. Accordingly, pharmacological and genetic studies indicated that GPR139 plays a role in the rewarding and analgesic effects of addictive drugs like alcohol and opioids [28, 29]. Molecularly, GPR139 has been shown to physically and functionally interact with the $\mu$-opioid system to coordinate and regulate neuronal activity where the receptors are co-expressed [28]. GPR139 has also been noted to co-express with dopamine D2 receptors (D2R) and influence its signaling properties [26]. Aside from this circumstantial evidence, no published studies have examined the role of GPR139 in neuropsychiatric related behaviors and its contribution to brain neurophysiology remains largely unexplored.

In this study, we present the first comprehensive evaluation of GPR139's role in behavior. Using GPR139 knockout mice, we report a prominent role for this receptor in motor activity and operant behavior. Remarkably, we observed that loss of GPR139 results in several behavioral deficits seen in psychosis models: severe deficits in pre-pulse inhibition, social interactions and acoustic startle conditioning. Mice lacking GPR139 also display spontaneous head twitches normally observed in response to hallucinogenic drugs. Importantly, the behavioral anomalies can be pharmacologically rescued by either haloperidol or naltrexone with no effect on wild type mice, which is consistent with hyperactive dopaminergic and opioidergic control driving the behavioral deficits in mice lacking GPR139.

\section{MATERIALS AND METHODS}

Animals

Gpr139-1- mouse strain (GPR139tm1.1(KOMP)Vleg) on pure C57BL/6N background was generated from embryonic stem cell clone 10338B-A5, created by Regeneron Pharmaceuticals Inc. and obtained from KOMP repository at the University of California, Davis. Mice evaluated in this study were littermates obtained through crossing heterozygous parents. Animals were housed in groups (unless otherwise stated) in a temperature-controlled environment on a 12-h light-dark cycle (6:00 AM light cycle; 6:00 PM dark cycle) with food (Teklad Global $16 \%$ protein rodent diets; Envigo Inc., Wisconsin USA) and water ad libitum.

Groups were compiled to ensure minimization of factors (i.e., weight, sex, health). Mice were within $18-34 \mathrm{~g}$ in weight at start of all studies. All tested groups contained a control group and consisted of male and female mice. Males and females were tested on the same day but at different times so that they were not in the room at the same time. Animals were tested during the middle of the light cycle, except for the operant learning cohort, to avoid long term disruption of sleep cycles. Testing of animals for operant learning was done at the middle of the dark cycle (8:00 AM dark cycle; 8:00 PM light cycle). Only one session of testing per animal was completed each day except for habituation sessions for the social interaction test. All recordings were blinded prior to scoring. Experimental results that were not blinded were collected by program software. Program software was calibrated ( 30 min before the start of sessions. No animals were excluded prior to and during studies since all were healthy. All animals were group housed, except for the operant learning group due to food restrictions. The operant learning cohort was individually housed and given 1 week for acclimation. Weight, behavior and health of the cohort was monitored during acclimation. All animals maintained weight, good health, and behavior during and after acclimation. All studies were carried out in compliance with the National Institute of Health guidelines. All procedures were reviewed and approved by the IACUC committee at the Scripps Research Institute.

\section{Open field}

Mice ( $n=8-15$ per group; either 2-3 or 5-6 months old, males and females) were placed into the center of an open field arena
$(140 \mathrm{~cm} \times 140 \mathrm{~cm} \times 140 \mathrm{~cm})$ and monitored (ANY-Maze, Stoelting Co., Illinois, USA) for $2 \mathrm{~h}$. Mouse locomotor activity, position in the cage and stereotypic behaviors were recorded by video camera. Habituation was assessed by comparing the reduction in locomotor activity between the initial and final 10-min interval.

\section{Evaluation of head twitching}

Mice ( $n=11-15$ per group; 4 months old, males and females) were placed individually into sterile, empty housing cages and recorded for $30 \mathrm{~min}$. High speed video recordings (C920 1080p, Logitech Int., Lausanne, Switzerland) were double blinded and abnormal rhythmic side to side head movements (head twitches) were counted over 30 min. Each head twitch was counted as one and scored using the automated feature recognition algorithm in Ethovision XT software (Noldus Information Technology Inc., Virginia, USA).

\section{Novel object recognition}

Animals ( $n=13-17$ per group; 3-4 months old, males and females) were habituated in an empty open field arena (140 $\mathrm{cm} \times 140 \mathrm{~cm} \times 140 \mathrm{~cm}$ ) for 5 -min sessions twice per day with $4 \mathrm{~h}$ between each session for 3 days. Subjects were then placed back into the arena containing two identical objects and allowed to explore for $10 \mathrm{~min}$. Animals were returned back to the arena the following day with one familiar object and a novel one. Mice were allotted $10 \mathrm{~min}$ for exploration. All sessions were tracked (ANYMaze, Stoelting Co., Illinois, USA) for movement and time spent exploring objects.

\section{Social interaction test}

Animals ( $n=13-17$ per group; 3-4 months old, males and females) were placed into a 3-chamber apparatus. Each chamber $(30 \mathrm{~cm} \times 30$ $\mathrm{cm} \times 30 \mathrm{~cm}$ ) contained dividing walls with an open middle section to allow for access. Both outer chambers contained wire cups. Mice were given free access to the apparatus for 5 min (absent of other mice) to habituate and confirm initial unbiased preference. To test for sociability, mice were placed into the middle chamber of the apparatus with one outer chamber containing one mouse confined in wire cup and the other chamber containing an empty wire cup. For social novelty preference, mice were again placed into middle chamber with one chamber containing the familiar mouse and the other containing the novel mouse confined in wire cups. The sex of familiar and novel mice introduced for assayin social interacitons matched the sex of the test subject: males were paired with males and females with females. Mice were allowed $10 \mathrm{~min}$ to explore all chambers in both tests. Time spent in each chamber was recorded (ANY-Maze, Stoelting Co., Illinois, USA).

\section{Acoustic startle response and pre-pulse inhibition}

Animals ( $n=15-19$ per group; 4-5 months old; males and females) were placed into an acoustic startle/PPI apparatus (SRLab, San Diego Instruments). The apparatus was calibrated for sound and startle amplitude prior to the start of each session. During the initial session, mice underwent numerous presentations of $120 \mathrm{~dB}$ white noise for $40 \mathrm{~ms}$, and the startle response was recorded. Animals were returned to the apparatus on the following day for pre-pulse inhibition. Mice were exposed to $120 \mathrm{~dB}$ white noise for $40 \mathrm{~ms}$ followed by a $20 \mathrm{~ms}$ pre-pulse of either 4,8 or $16 \mathrm{~dB}$ above background noise, or no stimulus. Startle response was again recorded.

\section{Cued fear conditioning}

Cued fear conditioning was performed using a paradigm adapted from previously published studies [30]. Mice ( $n=13-15$ per group; $\sim 5$ months old; males and females) were handled for 3 days prior to conditioning habituation. During habituation, animals were placed into fear conditioning chambers (Noldus Information Technology Inc., Virginia, USA) and left for 4 min with no shock delivered and with no stimulus noise. Habituation was repeated 
3 times for each mouse, all were 4-min exposures to the chamber without any stimulus and done on consecutive days. Animals were returned to their home cage and housing room after each session. During cued fear conditioning training, mice were placed into chambers and received one shock every $90 \mathrm{~s}$ for a total of 3 foot shocks. Prior to foot shocks, white noise stimulus was emitted for $30 \mathrm{~s}$. Mice were tested for learned association 1 day and 1 week after the training session. During these testing sessions, mice were placed into the chamber, but no noise and no shock was performed. The amount of time spent freezing and the total movement was recorded (Noldus Information Technology Inc., Virginia, USA).

\section{Water maze visual learning}

Mouse vision was assessed by spatial navigation in a water maze where animals ( $n=9-10$ per group; $\sim 6$ months old; males and females) were trained to find a visible escape platform while swimming. The protocol was previously described in detail [31]. Briefly, animals were individually placed into a water maze that was $\sim 1.2 \mathrm{~m}$ in diameter in a bright photopic $\left(100 \mathrm{~cd}^{*} \mathrm{~m}^{2}\right)$ environment and tracked using EthoVision XT (Noldus Information Technology Inc., Virginia, USA). Uniform room luminance settings were stably achieved by an engineered adjustable light-source and constantly monitored with a luminance meter LS-100 (Konica Minolta). Animals were given 5 daily trials with visible but variable platform locations for 3 consecutive days and the time to reach the platform was recorded and averaged for each day. The platform was placed pseudo-randomly in the water tank and all external visual cues were eliminated. To ensure that escape time on the platform requires vision, in separate trials the platform was hidden (submerged) and the time to randomly encounter the invisible platform was measured in 5 trials.

\section{Operant conditioning}

Conditioning with food reward was used to evaluate general operant responsiveness of animals in a setting requiring processing sensory information and engaging learning and memory functions. The methodology has been established and described previously $[32,33]$. Naïve mice $(n=10-12$ per group; $\sim 4$ months old at start of first session; males and females) were placed into operant conditioning chambers (Med Associates Inc., Maine USA) and were trained to differentiate between two levers: one paired with a food reward and the other with no consequences (FR5TO20). All subjects were required to meet criteria (FR5TO20; at least 20 rewards obtained in an hour session; 85\% accuracy between levers).

Once mice were capable of obtaining rewards on consecutive days, they were given 1 week without operant training and remained in home cages during this time period. After 1 week, they were placed back into the conditioning chambers and retrained for lever pressing with food reinforcement.

\section{Drug treatments}

Animals ( $n=6$ per group; 3-6 months old; males and females) were assessed for locomotor activity, head twitching, pre-pulse inhibition and novel object recognition. The same animals received both control treatment (saline) and after a 2-3 day (head twitching and pre-pulse) or a 1-week break, they were injected with naltrexone $(10 \mathrm{mg} / \mathrm{kg}$, s.c.; Selleck Chemicals LLC, Pennsylvania, USA) or haloperidol $(0.2 \mathrm{mg} / \mathrm{kg}$, s.C.; Selleck Chemicals LLC, Pennsylvania, USA) immediately prior to testing. Both groups were also administered saline as vehicle control. Animals were given a one-week recovery period between individual behavioral testing sessions.

Thallium flux assay

HEK293T cells were maintained in DMEM with $10 \%(\mathrm{v} / \mathrm{v})$ fetal bovine serum, non-essential amino acids, and $1 \mathrm{mM}$ sodium pyruvate. Transfections were performed as described using Lipofectamine 3000 (Invitrogen, Carlsbad, CA) [25]. Briefly, the following amounts of CDNAs were transfected: $0.84 \mu \mathrm{g}$ Dopamine-2 Receptor pcDNA3.1, $0.42 \mu \mathrm{g}$ HASP-HA-GPR139 pcDNA3.1, $1.26 \mu \mathrm{g}$ GIRK-1-AU5 pCMV5 and $1.26 \mu \mathrm{g}$ mGIRK-2a pCMV5, $0.84 \mu \mathrm{g}$ rat Gao pCMV, $0.42 \mu \mathrm{g} \mathrm{G \beta 1}$ pCDNA3.1, $0.42 \mu \mathrm{g} \mathrm{G \gamma 2}$ pcDNA3.1. Cells were incubated for $18-24 \mathrm{~h}$ at $37^{\circ} \mathrm{C}$ and $5 \% \mathrm{CO}_{2}$.

Thallium flux assays using the Molecular Devices FLIPR ${ }^{\circledR}$ Potassium Assay Kit were performed as described [25]. Measurements were made on the FLIPR Tetra ${ }^{\circledR}$ system. Basal signaling was measured for 10 reads at $0.1 \mathrm{~s}$ per read. Ten $\mu$ of $5 x$ compounds was then added to $40 \mathrm{ul}$ of cell culture and the plates were read for the next $200 \mathrm{~s}$. Data were normalized as fold over baseline and the change in activation over time was compared by fitting a straight line to the data within the initial linear range of activation (15-25 s) using GraphPad Prism 8.

\section{HiBiT cell surface abundance}

HEK293T cells were transfected as in the thallium flux assay, but the Dopamine- 2 receptor was modified amino-terminally with a $\mathrm{HiBiT}$ tag. One day after transfection, cells were re-seeded into 96well clear-bottom white plates at $1 \times 10^{5}$ cells per well in $100 \mu \mathrm{l}$ HEK293T maintenance media without phenol red. Cells were incubate $3-4 \mathrm{~h}$ at $37{ }^{\circ} \mathrm{C}$ and $5 \% \mathrm{CO}_{2}$. Fifty $\mu \mathrm{l}$ of overlaying media was removed. HiBiT Extracellular Detection reagent (Promega) was prepared by diluting HiBiT Extracellular Substrate 1:100 and LgBiT Protein 1:50 in BRET buffer (1x phosphate-buffered saline, $0.5 \mathrm{mM}$ $\mathrm{MgCl}_{2}$ and $0.1 \%(\mathrm{w} / \mathrm{v}) \mathrm{D}$-glucose). Fifty $\mu \mathrm{l}$ of detection reagent was pipetted into $50 \mu \mathrm{l}$ of cell media and incubated for $2 \mathrm{~min}$. Luminescence was measured kinetically using the Perkin Elmer Envision Plate Reader until a maximum signal was reached.

Statistical analysis

Statistical analysis was done using GraphPad Prism 8.4.3 software. For behavioral evaluation direct comparisons between genotypes for open field stereotypy, head twitches, acoustic startle response and operant learning results were analyzed using two tailed, unpaired Student's $t$ test with 95\% confidence level. Prepulse inhibition and cued fear conditioning was analyzed using two-way ANOVA with Bonferroni multiple comparison test comparing means between genotype during same decibel and/or sessions. Social interaction test results were analyzed with one-way ANOVA followed by Tukey's multiple comparison test with the mean of each column within the testing same session. Novel object test was analyzed with two-way ANOVA Bonferroni multiple comparison test between means in the same session.

Cohorts treated with vehicle and drugs were analyzed using two-way ANOVA Bonferroni multiple comparison test between means of the same genotype to observe the effects of the drug during open field, head twitches, prepulse inhibition and novel object recognition.

Functional assays in transfected cells were conducted across 4 independent experiments, each with 3 technical replicates. The results were analyzed by unpaired two tailed Student's $t$ test. The $p$ values reported throughout the study had the following designated thresholds: ${ }^{*} p<0.05,{ }^{* *} p<0.01,{ }^{* *} p<0.001$ and $* * * * p<0.0001$.

\section{RESULTS}

Loss of GPR139 induces late-onset hyperactivity and stereotypic head twitches in mice

We began examining the role of GPR139 in neuropsychiatric processes by evaluating mouse behavior in the open field. Our previous studies with younger mice revealed no significant changes in overall locomotor activity associated with the loss of GPR139 ([28]; Fig. 1A). However, older Gpr139 ${ }^{-1-}$ mice showed significant hyperactivity as compared to their wild-type Gpr139 $1 /+$ 
littermates (Fig. 1A). The increase in locomotor activity was evident from the initial period and lasted throughout the entire time of investigation resulting in a substantial increase in the total distance traveled (Fig. 1B). Both genotypes exhibited a similar level of habituation to a novel environment (Fig. 1C).

Interestingly, despite increased levels of activity, we observed that Gpr139 $9^{-/-}$mice spend more time immobile (Fig. 1D). This was accompanied by increased movement speed during the active state (Fig. 1E) indicating that mice lacking GPR139 displayed prolonged bouts of inactivity interspersed with periods of elevated ambulation. We further observed that $G p r 139^{-1-}$ mice displayed increased stereotypic rotations (Fig. 1F) and exhibited anxiolytic-like behavior including reduced thigmotaxis (Fig. 1G) and increase in the time spent in the center (Fig. 1H). Interestingly, we noticed that $G$ pr139-/- mice also exhibited another striking phenotype - spontaneous head twitches, a behavior typically observed in response to hallucinogenic drug administration. Gpr $139^{+/+}$control littermates displayed very minimal or no head twitches (Fig. 1I). In summary, these data indicate that loss of GPR139 induces late-onset hyperactivity accompanied by changes in the structure of the activity pattern suggestive of accompanying behavioral disruptions.

\section{Prominent sensorimotor deficits in mice lacking GPR139}

We next examined how loss of GPR139 influences responses of mice to external stimulation. First, we examined acoustic startle responses (Fig. 2A). When naïve mice were exposed to an abrupt acoustic stimulus $(120 \mathrm{~dB})$, both genotypes exhibited similar startle response upon the initial exposure, suggestive of normal reaction and lack of auditory impairment (Fig. 2B). In order to assess the ability of the mice to integrate sensory information, they were then exposed to varying intensities of conditioning stimuli delivered $40 \mathrm{~ms}$ prior to the startle-inducing stimulus (Fig. 2A). As expected, delivery of this "pre-pulse" strongly attenuated the acoustic startle response of $\mathrm{Gpr} 139^{+/+}$mice in a stimulus intensity-dependent manner (Fig. 2C). In contrast, Gpr139-/- mice had prominent deficits in the development of the pre-pulse inhibition showing virtually no conditioning to the stimulus even at the highest intensity range (Fig. 2C). Together, these data indicate that loss of GPR139 compromises the ability of mice to integrate sensory information with motor responses.

Next, we assessed the ability of the mice to integrate sensory cues with an aversive stimulus (foot shock) in a fear conditioning assay (Fig. 2D). Prior to conditioning, naïve mice of both genotypes showed a similarly low level of freezing behavior when introduced into the chamber (Fig. 2E). When auditory cue paired footshocks were repeatedly delivered during training, Gpr $139^{-1-}$ mice showed an increase in freezing behavior, indistinguishable from the Gpr $139^{+1}$ + littermates (Fig. 2E). However, when subjects were tested $24 \mathrm{~h}$ later in a different context while providing the same auditory cue without foot shocks, Gpr139-/- mice displayed substantially lower freezing relative to Gpr139 $9^{+/+}$mice. A week later, the response in Gpr139+/+ mice was diminished, reflecting the extinction of the association; however, Gpr139-/- mice remained at the same level as day 1 after training (Fig. 2E). These alterations in fear conditioning responses are indicative of significant disruptions in associative leaning which requires integration, consolidation and retention of sensory information.

To test selectivity of these deficits we assessed general cueguided spatial navigation of mice and their visual function in the water maze task (Fig. 2F, G). Gpr139 ${ }^{-1-}$ mice were indistinguishable from their Gpr139+/+ littermates in the ability to locate the visible escape platform similarly improving the performance with each consecutive session (Fig. $2 \mathrm{H}, \mathrm{I}, \mathrm{J}$ ). At the end of the trials we ensured that vision was required for achieving the short escape times in trained mice by conducting a control session with a hidden platform. This experiment showed that it takes mice of both genotypes significantly longer to locate platform randomly.
These results confirm that Gpr139-/- mice have intact vision and spatial navigation abilities. Therefore, the deficits in sensorimotor integration that we observe in mice lacking GPR139 are selective.

Delayed acquisition of operant responsiveness in mice lacking GPR139

To further study the impact of sensorimotor impairments, we analyzed the ability of Gpr139-1- mice to engage in complex behaviors. We employed an operant paradigm to examine the acquisition of lever pressing in response to food reinforcement (Fig. 3A). In this task, mice escalated their pressing on active, but not inactive, levers in order to obtain a food reward at a fixed ratio of 5 and time out of $20 \mathrm{~s}$ (Fig. 3B). Gpr $139^{+/+}$mice took $\sim 7$ days to reach stable lever pressing behavior at the level of 200 presses in a session. In contrast, Gpr139-/- mice reached the same criterion level of 200 active lever presses per session after $\sim 17$ sessions of gradually increasing their operant responsiveness (Fig. 3C). When quantified for the duration of the experiment, Gpr139-/- mice had significantly fewer active lever presses than Gpr139+/+ (Fig. 3D) but showed substantially increased activity at the inactive lever (Fig. 3E). Furthermore, Gpr139-/- displayed an inability to discriminate between the levers during early acquisition sessions (Fig. 3F). Once all animals met criteria and stabilized lever responses for 3 consecutive days, they were returned to home cages to undergo inactive extinction. After 1 week of inactive extinction, animals were placed back into operant chambers and reinstatement was assessed. Again, Gpr139-/- mice showed substantially slower return to the stable lever pressing as compared to Gpr139+/+ controls (Fig. 3B). In summary, we conclude that loss of GPR139 significantly delays, but does not prevent, operant responsiveness to reinforcement with food.

GPR139 null mice feature deficits in subject and object recognition To further probe the ability of mice lacking GPR139 to discriminate and respond to external cues, we studied their social interaction behavior, frequently compromised in neuropsychiatric models especially when the sensorimotor integration is compromised, e.g., in schizophrenia models. In the sociability test (Fig. 4A) when wild-type littermate mice were given a choice to interact with an empty space or a space containing a mouse, they showed a clear preference for a social context (Fig. 4B). During the next session $24 \mathrm{~h}$ later, when a new mouse was introduced to the chamber, Gpr139 $9^{+/+}$mice showed a preference for a social novelty, interacting more with a new mouse relative to a familiar one (Fig. 4B). This behavior was drastically different for the Gpr139-/mice, which showed no preference for the mouse in the sociability test and no preference for the novel subject in the social novelty setting (Fig. 4C).

In order to test whether deficits in social interactions are specifically related to impairment in social preferences or a more general inability to discriminate features in the environment, we performed a novel object recognition test (Fig. 4D). In this paradigm, wild-type mice showed significant preference for a new object introduced to replace the familiar object to which the mice were habituated (Fig. 4E). In contrast, Gpr139 ${ }^{-1-}$ mice showed no preference for the novel object and spent an equivalent amount of time exploring the new object as they did exploring the familiar one (Fig. 4F). Furthermore, the time spent interacting with novel vs. familiar objects during the second session was no different from the time the Gpr139-/- mice interacted with two identical objects in the first session (Fig. 4F). Together, these results indicate that mice lacking GPR139 manifest severe deficits in the ability to differentiate between animals as well as between inanimate objects.

Antagonism of opioid signaling reverses several behavioral deficits in mice lacking GPR139

In searching for the neurobiological underpinnings of the behavioral deficits observed in $\operatorname{Gpr} 139^{-/-}$mice, we considered 
A

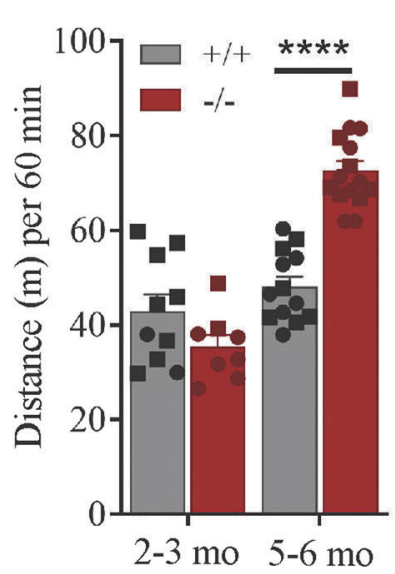

D

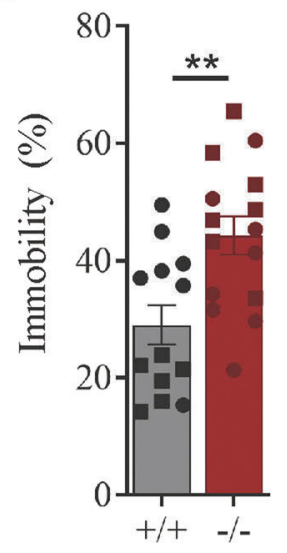

G

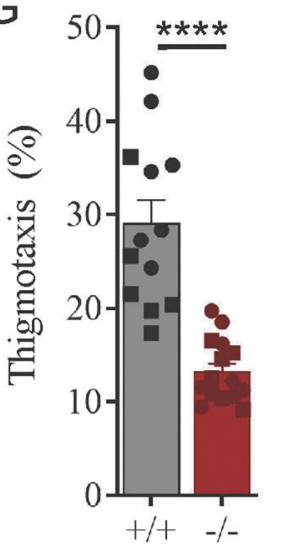

B

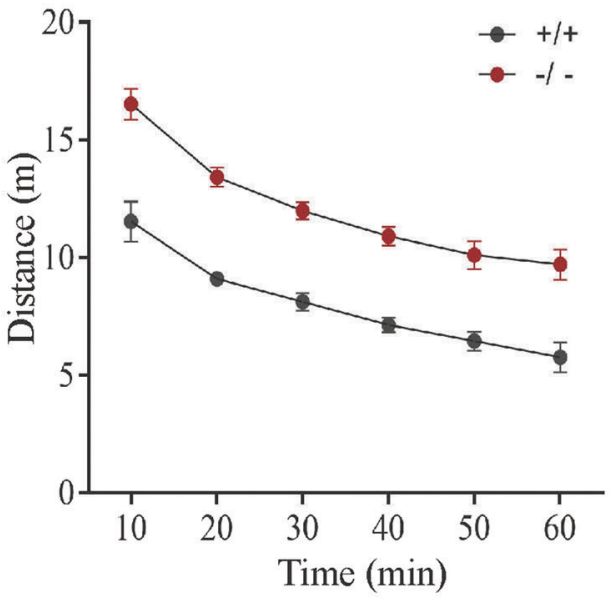

E

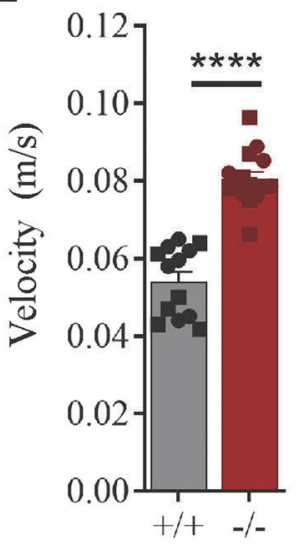

$\mathrm{H}$

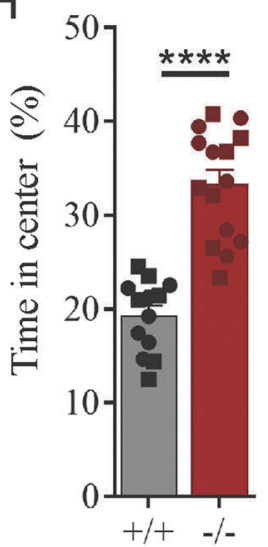

C

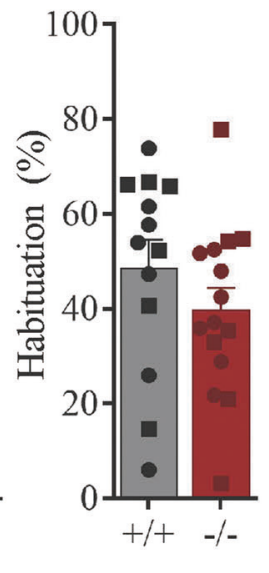

$\mathrm{F}$

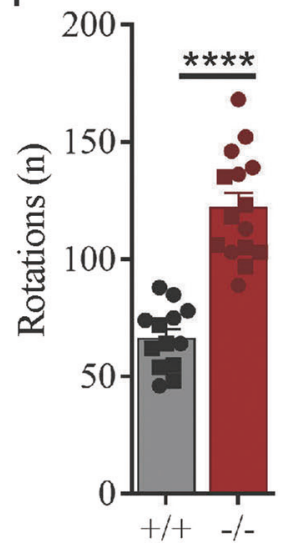

I

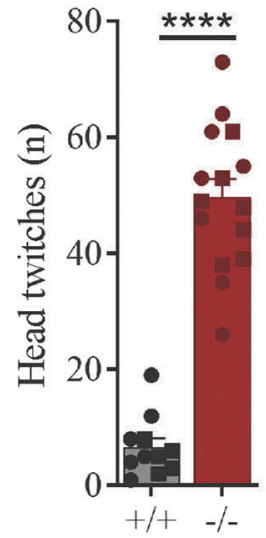

Fig. 1 Evaluation of mouse behavior in the open field. A Cumulative locomotor activity of mice during 60 min of observation. Unpaired $t$-test of $2-3$ months old males $p=0.3017 ; 5-6$ months old males $p=0.0001 ; 2-3$ months old females $p=0.2502 ; 5-6$ months old females $p<$ 0.0001 . B Changes in locomotor activity as a function of time following placement in the open field chamber. Two-Way ANOVA analysis of male mice: interaction $p=0.1092$; time $p<0.0001$; genotype $p=0.0004$. Two-Way ANOVA analysis of female mice: interaction $p=0.9573$; time $p<0.0001$; genotype $p<0.0001$. C Quantification of reduction in locomotor activity during the observation time. Unpaired $t$-test analysis of males $p=0.1086$; females $p=0.4685$. D Quantification of time mice spent immobile. Unpaired $t$-test of males $p<0.0001$; females $p=$ 0.7324 E Quantification of movement speed during the active movement bouts. Unpaired $t$-test of males $p=0.0002$; females $p<0.0001$. F Quantification of rotations. Unpaired $t$-test of males $p<0.0001$; females $p=0.0002$. G Analysis of relative time mice spent close to the walls of the chamber. Unpaired $t$-test of males $p=0.0028$; females $p<0.0001$. $\mathbf{H}$ Analysis of time mice spent in the center of the chamber. Unpaired $t$-test of males $p=0.0015$; females $p<0.0001$. I Tracking of spontaneous involuntary head twitching in mice placed in an empty cage. $11-15$ mice of both sexes were used. Unpaired $t$-test of males $p<0.0001$; females $p<0.0001$. In all panels statistical analysis was performed combining both sexes and significance was designated as: ${ }^{*} p<0.05,{ }^{* *} p<0.01,{ }^{* * *} p<0.001$ and ${ }^{* * *} p<0.0001$, data sets (mean \pm SEM) analyzed using unpaired two tailed Student's $t$ test. In bar graphs only, male data are in squares, female - circles. Plots in panel B account for both sexes. 
A

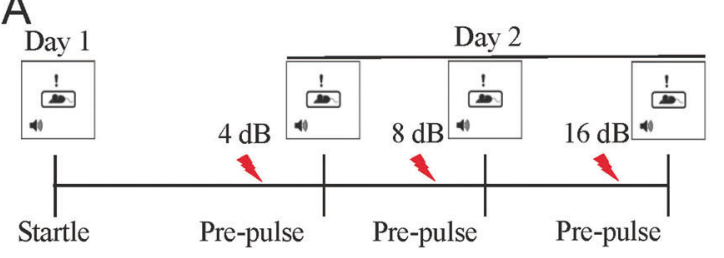

$\mathrm{B}$

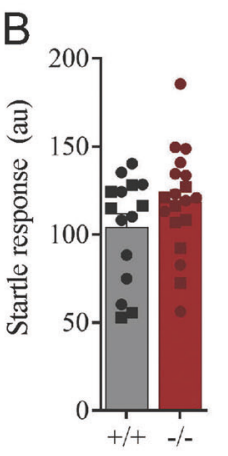

C

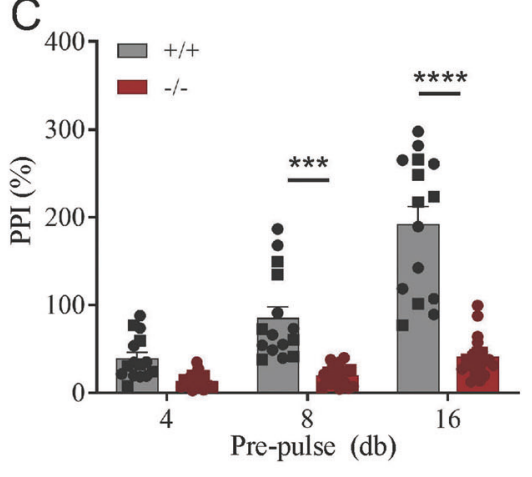

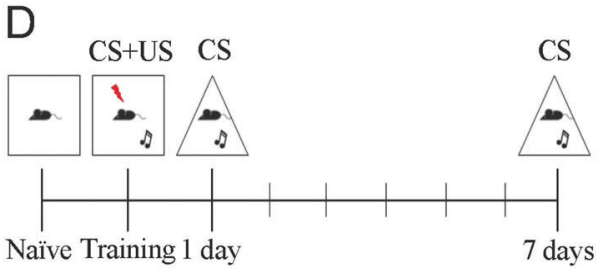

E

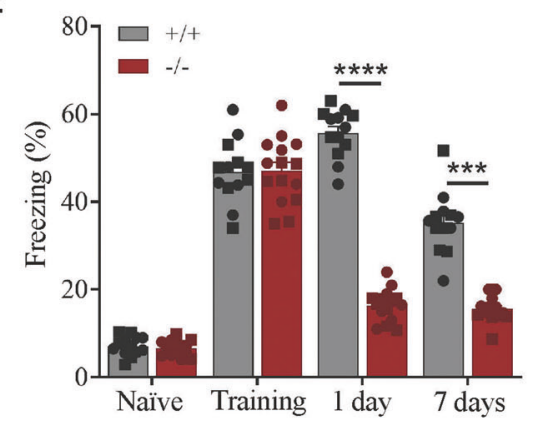

$\mathrm{F}$

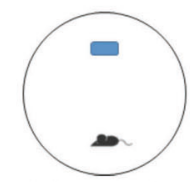

Visible Platform

(Trial 1-3)

$H$

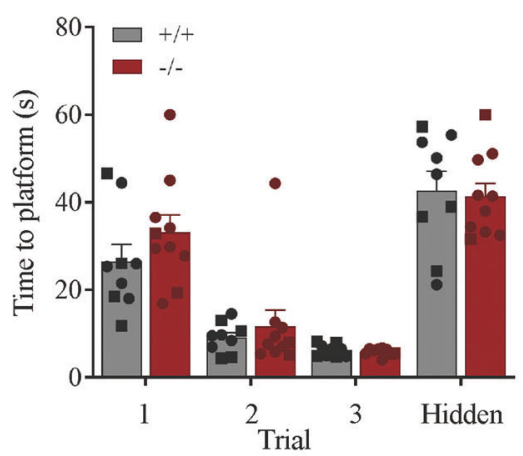

G

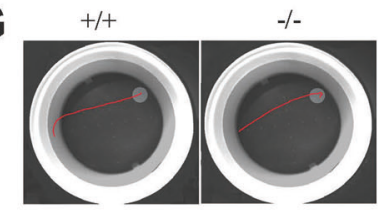

Visible (trial 3)

|

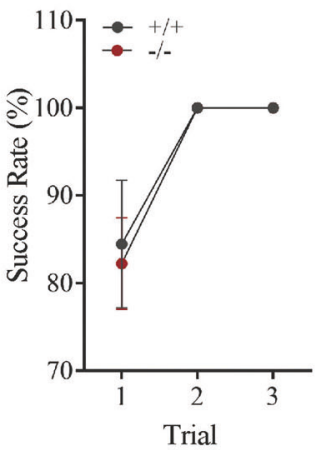

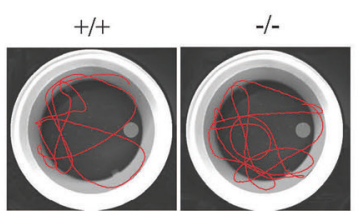

Hidden

J

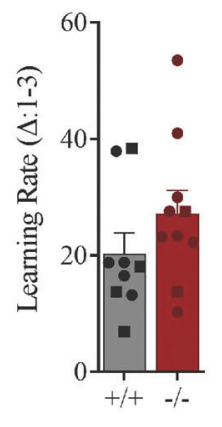

Fig. 2 The effect of GPR139 loss on sensorimotor integration, vision and spatial navigation. A Schematic of the timeline and design of experiments for evaluating mouse behavior in acoustic startle and pre-pulse inhibition paradigms. B Acoustic startle responses of naïve mice presented with $120 \mathrm{~dB}$ stimulus. Unpaired $t$-test of males $p=0.6223$; females $p=0.2527$. C The effect of delivering conditioning pre-pulses of varying intensities on the startle responses of mice. Two-Way ANOVA analysis of male mice: interaction $p<0.0001$; dB $p<0.0001$; genotype $p=0.0008$. Two-Way ANOVA analysis of female mice: interaction $p<0.0001 ; \mathrm{dB} p<0.0001$; genotype $p<0.0001$. D Schematic of the timeline and design of experiments for evaluating mouse behavior in cued fear conditioning experiments pairing white noise conditioning stimulus (CS) with electric shock unconditional stimulus (US). E Analysis of mouse behavior during various phases of fear conditioning. Two-Way ANOVA analysis of male mice: interaction $p<0.0001$; time $p<0.0001$; genotype $p<0.0001$. Two-Way ANOVA analysis of female mice: interaction $p<0.0001$; time $p<0.0001$; genotype $p<0.0001$. F Schematic of water maze experiment. G Swimming tracks of representative animals during sessions with visible and hidden platforms. $\mathbf{H}$ Analysis of platform finding latencies. Trial 1 to 3 was analyzed using Two-way ANOVA: interaction $p=0.3498$; time $p<0.0001$; genotype $p=0.3098$. Hidden trial was analyzed using unpaired $t$-test $p=0.8054$. I Analysis of the success rate in visible platform finding task. Two-way ANOVA with Bonferroni's multiple comparison test: males interaction $p=0.9037$, trial $=0.1846$, genotype $p=0.4609$; females interaction $p=0.5781$, trial $p=0.0129$, genotype 0.4115 . J Analysis of the learning rate defined as difference in escape latencies between session 1 and session 3. Unpaired $t$-test of males $p=0.9748$; females $p=0.1752$. In all panels statistical analysis was performed combining both sexes and significance was $* * * p<0.001$ and ${ }^{* * *} p<0.0001$, data sets (mean \pm SEM) as analyzed using unpaired two tailed Student's $t$ test (panel B) and two-way ANOVA with Bonferroni's multiple comparison test for panels $\mathbf{C}$, E, and $\mathbf{H}$ for trials 1-3. In bar graphs only, male data are in squares, female - circles. Plots in panel I account for both sexes.

the reports that loss of GPR139 substantially augments the signaling via $\mu$-opioid receptors (MOR) [28]. In order to determine whether enhancement of opioidergic signaling contribute to the behavioral manifestations observed in Gpr139-/- mice, we evaluated the effects of MOR pharmacological blockade. Administration of MOR antagonist naltrexone exerted profound behavioral effects selectively in Gpr139-/- mice. We found that naltrexone substantially reduced head twitching in Gpr139-1mice without noticeable effects on control Gpr139 $9^{+/+}$littermates (Fig. 4G). Strikingly, Gpr139 $19^{-/-}$mice treated with naltrexone also gained an ability to discriminate between novel and familiar object, a property that was completely absent in a control group 
A

Acquisition

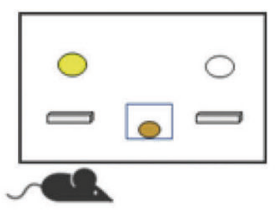

Sessions 1-22

(FR5TO20)
Extinction

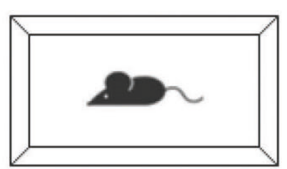

Sessions 23-29

(Home cage)

\section{Reinstatement}

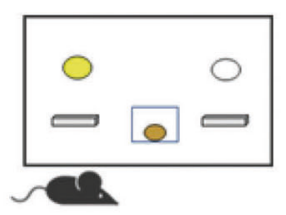

Sessions 30-37

(FR5TO20)

B

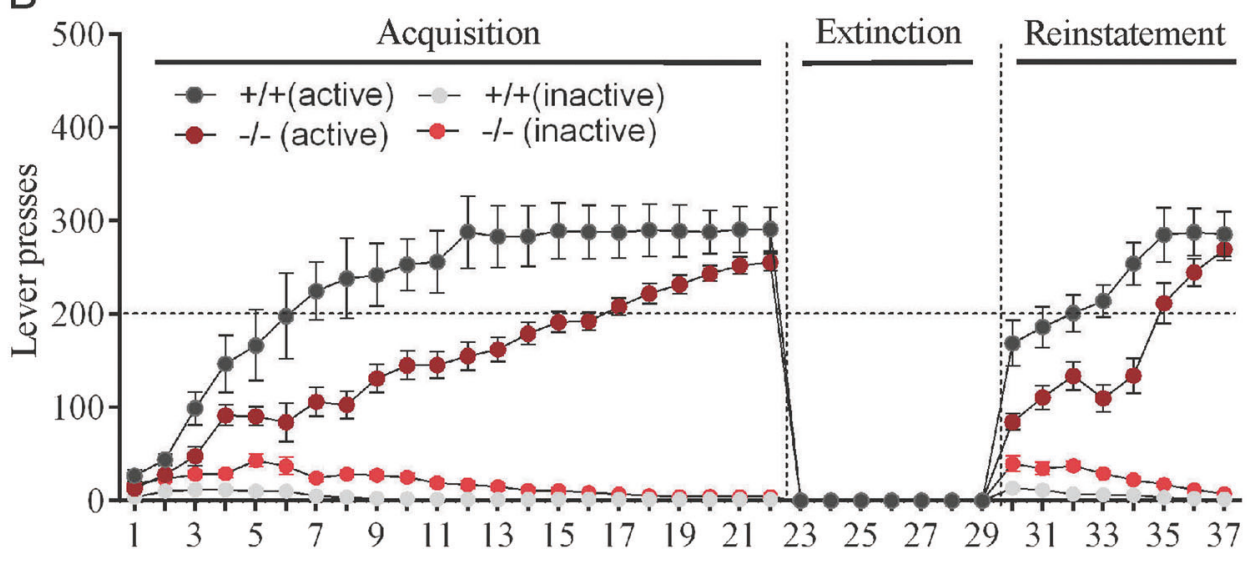

Session
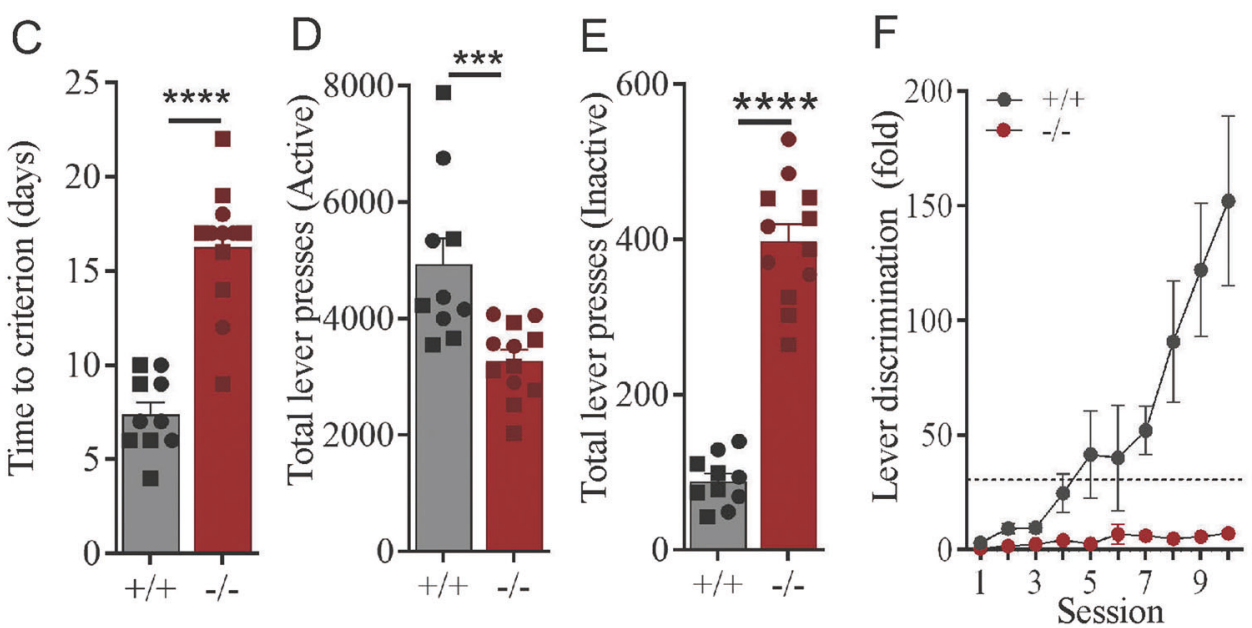

Fig. 3 Operant behavior of mice lacking GPR139. A Schematic of the experimental design evaluating mouse behavior in operant task. Mice were rewarded for pressing the active levers with food. B Dynamics of lever pressing behavior of mice during different phases of operant food self-administration paradigm. C Quantification of duration of training to reach criterion of 200 lever presses per session. Unpaired $t$-test of males $p<0.0001$; females $p=0.0002$. D Quantification of total active lever presses for the duration of the entire acquisition phase (22 days). Unpaired $t$-test of males $p=0.0252$; females $p=0.0476$. E Quantification of total inactive lever presses for the duration of the entire acquisition phase (22 days). Unpaired $t$-test of males $p<0.0001$; females $p<0.0001$. F Analysis of lever discrimination dynamics during the initial 10 session of training. Presses on active levers are divided by the number of presses on inactive levers and the values are plotted. Statistical analysis was performed by combining mice of both sexes $(10-12)$ together, ${ }^{* * *} p<0.001,{ }^{* * * *} p<0.0001$, data sets (mean \pm SEM) were analyzed using unpaired two tailed Student's $t$ test. In bar graphs only, male data are in squares, female - circles. Plots in panels B and F account for both sexes.

that received saline (Fig. 4H). Again, control $\mathrm{Gpr}_{139^{+/+}}$mice treated with naltrexone discriminated between familiar and novel object as did the mice that received saline injections (Fig. 4I). We thus conclude that behavioral deficits related to novel object recognition and head twitching observed upon loss of GPR139 are likely related to the excess of opiodergic signaling as they can be rescued by opioid receptor antagonism.
GPR139 antagonizes D2R signaling

Recent evidence suggest that in addition to MOR, GPR139 may also directly interact with the dopamine D2 receptor (D2R) [26]. Interestingly, D2R belongs to the same subfamily of $G_{i / o}$-coupled GPCRs as MOR. We have recently found that the major mechanism used by GPR139 to antagonize MOR is related to its ability to signal via $\mathrm{Ga}_{\mathrm{q} / 11}$ to oppose the $\mathrm{Ga}_{\mathrm{i} / \mathrm{o}}$ actions of MOR at its 

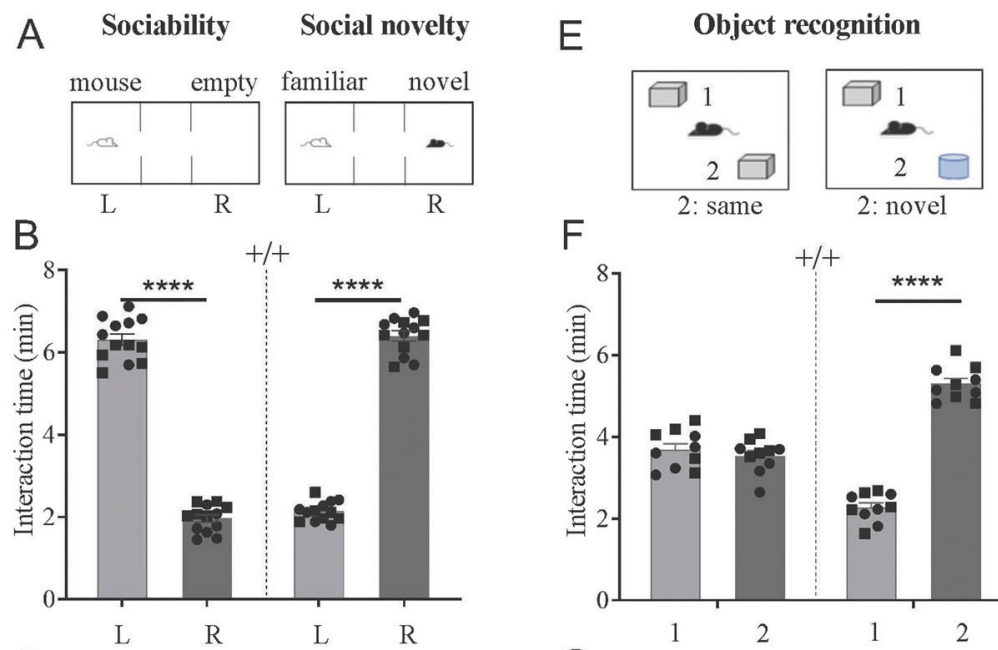

F
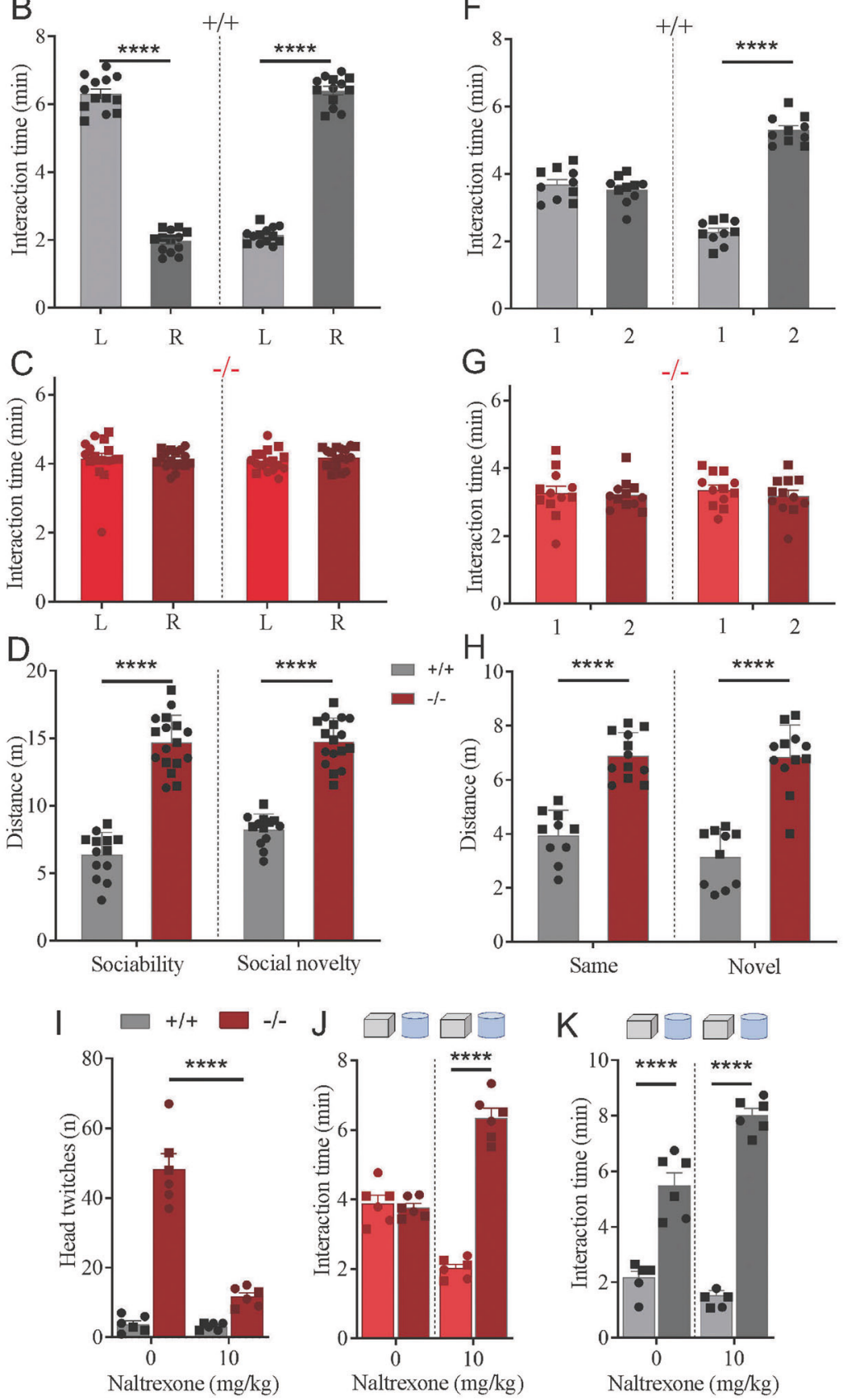

effectors [25]. Given the similar signaling mechanisms of D2R and MOR, we examined whether GPR139 can also antagonize signaling via $D 2 R$, the way it does for MOR. We used a cellbased assay that monitors D2R signaling by analyzing its ability to activate its canonical effector - $\mathrm{G}$ protein Inwardly Rectifying $\mathrm{K}^{+}$ (GIRK) channels. In this assay, opening of the GIRK channel is promoted by $\mathrm{G} \beta \gamma$ subunits released upon Gi/o activation by D2R and is determined by measuring the influx of thallium permeating specifically through the GIRK channels to increase fluorescence of a thallium-sensitive dye loaded into the cells (Fig. 5A).
Indeed, stimulation of cells transfected with D2R with dopamine resulted in robust increase in the rate of thallium influx (Fig. 5B). This effect was specific to D2R and cells transfected with GPR139 only did not respond to dopamine stimulation. However, coexpression with GPR139 prevented dopamine induced change in fluorescence mediated by D2R (Fig. 5B). Concentration response studies (Fig. 5C) further indicated that GPR139 markedly reduced the extent of the response ( 6-fold) defined by changes in maximal amplitudes (from $0.038 \pm 0.02 \mathrm{~s}^{-1}$ to $0.006 \pm 0.005 \mathrm{~s}^{-1}$ ) of the response and its sensitivity $(\sim 7$ fold $)$ defined by the 
Fig. 4 Impact of GPR139 ablation on social behavior, novel object recognition and rescue of behavioral deficits by opioid receptor blockade. A Schematic of the experimental design evaluating mouse behavior in sociability and social novelty paradigms. B Quantification of the time control Gpr139+/+ mice spent in chambers containing mouse or familiar mouse (L) and empty or novel mouse (R), respectively. The empty central chamber is marked as C. Unpaired $t$-test Gpr139 $9^{+/+}$males: sociability $p<0.0001$; social novelty $p<0.0001$. Unpaired $t$-test Gpr139 $9^{+/+}$females: sociability $p<0.0001$; social novelty $p<0.0001$. C Quantification of the time Gpr $139^{-1-}$ mice spent in chambers containing

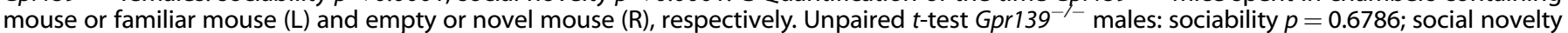
$p=0.8061$. Unpaired $t$-test Gpr $139^{-1}$ females: sociability $p=0.9101$; social novelty $p=0.1149$. D Analysis of locomotor activity during social interaction testing. Unpaired $t$-test sociability session males $p<0.0001$, females $p<0.0001$. Unpaired $t$-test social novelty session males $p<$ 0.0001 , females $p<0.0001$. E Schematic of the experimental design evaluating novel object recognition behavior. Mice were presented first with the same objects and the following day one object was replaced with a new one. F Quantification of the time control Gpr139 $9^{+/+}$mice spent interacting with familiar and novel objects. Unpaired $t$-test of Gpr139+/+ males: same object $p=0.7565$; novel object $p=0.0006$. Unpaired $t$-test of Gpr139 ${ }^{+/+}$females: same object $p=0.8781$; novel object $p<0.0001$. G Quantification of the time Gpr139 $9^{-/-}$mice spent interacting with familiar and novel objects. Unpaired T-test of Gpr139-1- males: same object $p=0.5357 ;$ novel object $p=0.4410$. Unpaired $t$-test Gpr139 ${ }^{-1-}$ females: same object $p=0.8063$; novel object $p=0.3138$. H Analysis of locomotor activity during novel object recognition testing. Unpaired $t$-test same object session males $p=0.0004$, females $p<0.0001$. Unpaired $t$-test novel object session males $p=0.0033$, females $p=0.0031$. I The effects of MOR blockade with naltrexone on spontaneous head twitching. J The effects of MOR blockade with

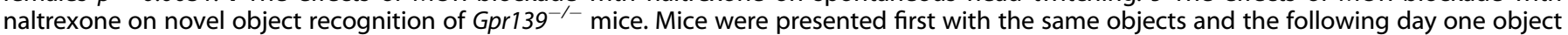
was replaced with a new one. Naltrexone was administered immediately prior to testing in both sessions. Light red: interaction with the familiar object (gray square); dark red: interaction with novel object (blue cylinder). $\mathbf{K}$ The effects of MOR blockade with naltrexone on novel object recognition of Gpr139 $9^{+/+}$mice. Mice were presented first with the same objects and the following day one object was replaced with a new one. Naltrexone was administered immediately prior to testing in both sessions. Light gray: interaction with the familiar object (gray square); dark gray: interaction with novel object (blue cylinder). In all panels statistical analysis was performed combining both sexes and significance was ${ }^{* * * *} p<0.0001$; data sets (mean \pm SEM) were analyzed using one-way ANOVA Tukey multiple comparison test for social interaction test, two-way ANOVA Bonferroni's multiple comparison test for novel objection recognition test and two-way ANOVA Bonferroni multiple comparison test within genotypes for naloxone rescue experiments. Male data are in squares, female - circles.

$\mathrm{EC}_{50}$ values (from $3.83 \pm 0.83 \mathrm{nM}$ to $23.15 \pm 8.47 \mathrm{nM}$ ) (Fig. 5D). We also found that co-transfection with GPR139 does not significantly influence the levels of D2R on the surface (Fig. 5E) suggesting that the inhibitory influence likely occurs downstream at the level of receptor signaling. Together, these results indicate that GPR139 can effectively counteract D2R function.

Antagonism of D2R signaling rescues many behavioral deficits in mice lacking GPR139

Given our observations that GPR139 inhibits D2R actions in vitro we next examined whether elimination of GPR139 in vivo could impact behavioral manifestations by enhancing dopaminergic signaling. This possibility was tested by evaluating the behavioral consequence of $\mathrm{D} 2 \mathrm{R}$ pharmacological blockade. Administration of a low dose $(0.2 \mathrm{mg} / \mathrm{kg})$ of the D2R antagonist haloperidol completely suppressed the locomotor hyperactivity of Gpr139-1mice without influencing the activity of $\mathrm{Gpr} 139^{+/+}$littermates (Fig. 5F). It also dramatically reduced the head twitching behavior

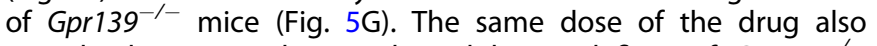
completely corrected pre-pulse inhibition deficits of Gpr139-1mice while having no effect on Gpr139+/+ littermates (Fig. 5H). Finally, haloperidol treatment was also able to rescue the novel object recognition deficits in $G$ pr139-/- mice bringing this behavior to the level seen in Gpr139+/+ littermates (Fig. 5I, J). Overall, we conclude that a spectrum of neuropsychiatric manifestations in mice lacking GPR139 arise from unrestrained opioidergic and dopaminergic signaling.

\section{DISCUSSION}

In this study, we present the results of a comprehensive behavioral evaluation of a mouse model with a global deletion of orphan receptor GPR139. We report that GPR139 knockout mice show many neuropsychiatric behavioral features including lateonset hyperactivity, lack of pre-pulse inhibition, loss in the ability to recognize novel objects, social interaction deficits, anxiolytic traits and compromised learning. Intriguingly, mice lacking GPR139 also displayed abnormal structure of their activity patterns reflected in irregular activity/rest intervals, patterns of space exploration, circling behavior and spontaneous head twitches. Altogether, these traits are suggestive of an altered perception of the environment and integration into actionable outcomes.
The combination of sensorimotor gating deficits, interaction with external stimuli/environment and cognitive deficits is reminiscent of psychosis symptoms in humans. Furthermore, several genetic mouse models sharing many behavioral features of GPR139 knockouts, in particular DISC1 mutant mice $[34,35]$ and mice with disruption in NGR1/ErbB4 [36, 37] are typically described as schizophrenia models [38]. In this context, a unique and highly interesting feature of the GPR139 knockout model is presented by the stereotypic head twitching. This behavior is usually induced by the drugs that target NMDA-type glutamate receptors and 5$\mathrm{HT} 2 \mathrm{~A} / \mathrm{C}$ serotonin receptors, e.g., PCP and $\operatorname{LSD}[39,40]$. Since these drugs are known to cause hallucinations in humans, head twitching in mice has been proposed to be a behavioral correlate of hallucinations in rodents [41]. Given that distorted perception of reality and hallucinations are cardinal features of schizophrenia $[42,43]$, the spontaneous head twitching in GPR139 knockouts could be further taken as a proxy for delusional behavior. Together, our behavioral observations suggest that loss of GPR139 in mice may indeed contribute to schizophrenia-like pathology. Although GPR139 has yet to be directly implicated in the pathology of schizophrenia, a recent genetic linkage study has also suggested a possible connection [44]. Our observations are further complemented by unpublished data about other schizophrenia-related behavioral alterations in another GPR139 null model [45] which apparently motivated evaluation of GPR139 agonist TAC-041 in mouse models and a human clinical trial for schizophrenia-related anhedonia [46, 47].

It is interesting to consider the observed neuropsychiatric phenotypes in GPR139 mice from the perspective of neuronal circuitry. GPR139 shows selective expression in distinct neuronal populations in the brain including specific neuronal ensembles in the limbic structures and connected nuclei [12]. Perhaps the most prominent is the expression of GPR139 in the medial habenula where it can even be considered a marker for this structure $[16,28,48]$. Habenula is an enigmatic nucleus involved in a range of processes including attention/arousal, reward and cognition modulating a number of neuromodulatory systems [49, 50]. Significantly, studies in humans note abnormalities in the habenula in patients diagnosed with schizophrenia [51, 52]. Furthermore, studies in rodents show alterations in the habenula of schizophrenia models $[53,54]$ and pharmacological/lesion manipulations of the habenula $[55,56]$ also produce some of the 
A

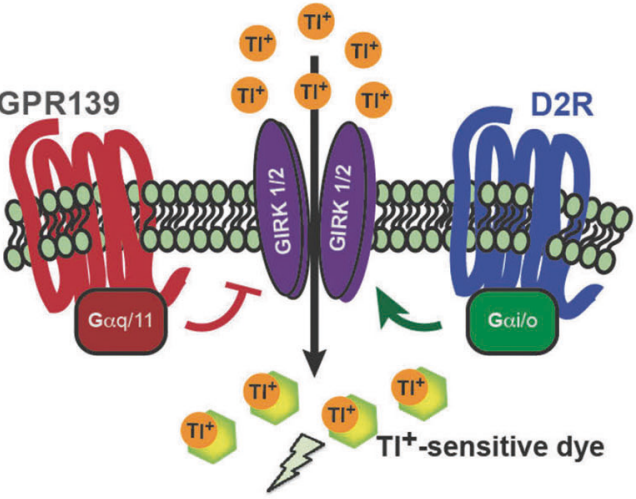

B

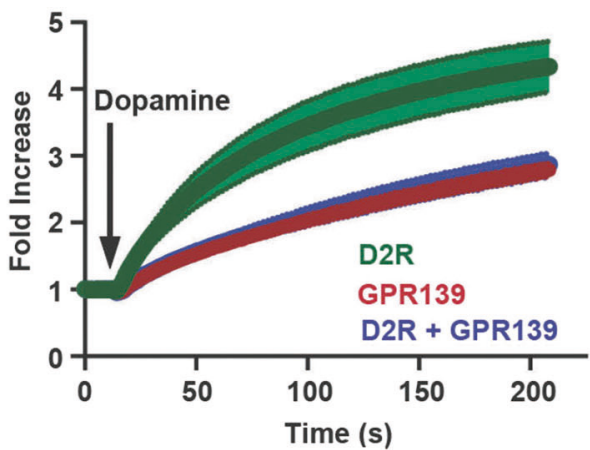

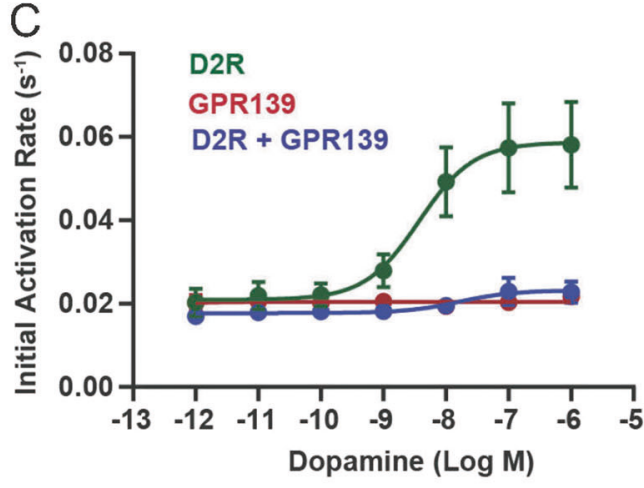

F

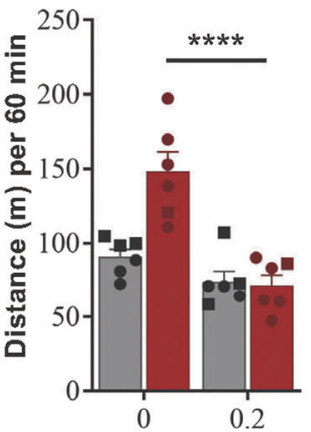

Haloperidol (mg/kg)
G

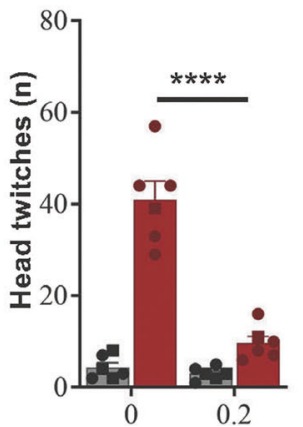

Haloperidol (mg/kg)

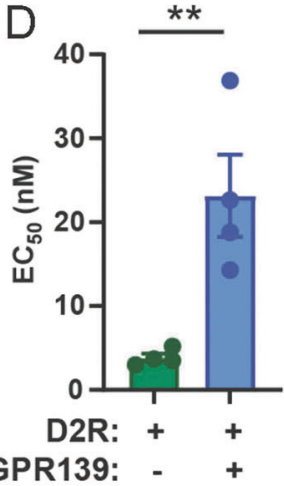

$\mathrm{H}$
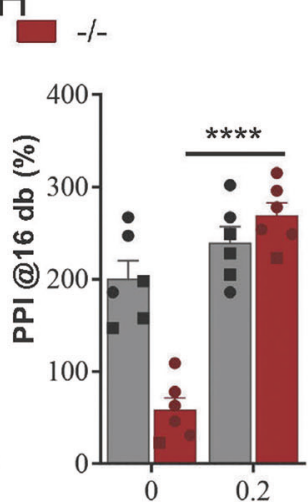

Haloperidol ( $\mathrm{mg} / \mathrm{kg}$ )
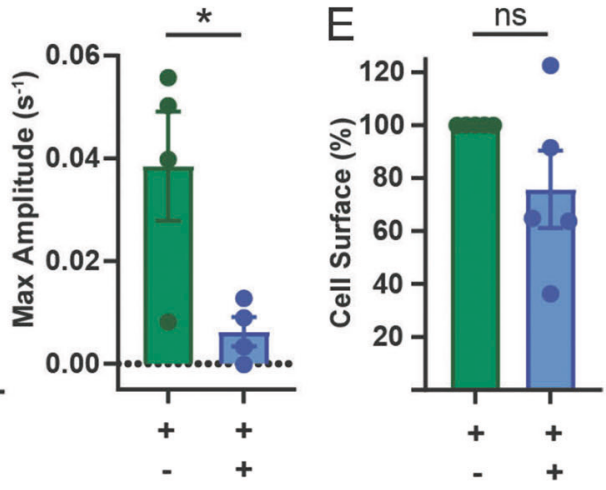

I

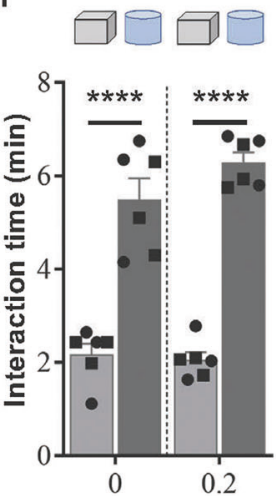

Haloperidol ( $\mathrm{mg} / \mathrm{kg}$ )

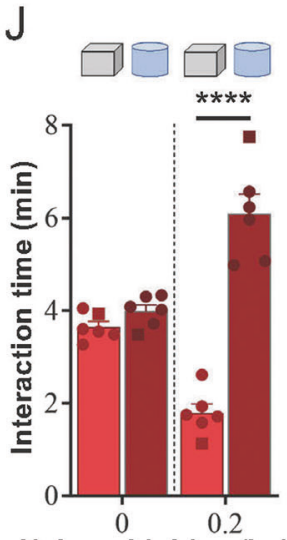

Haloperidol ( $\mathrm{mg} / \mathrm{kg}$ )

Fig. 5 GPR139 Inhibits D2R signaling and blockade of D2R signaling rescues many behavioral deficits in mice lacking GPR139. A Schematic of assay measuring GIRK channel activity by monitoring thallium flux. D2R signals via Gi/o to release G $\beta \gamma$ subunits that promote GIRK opening to result in acceleration of the thallium flux detected as a change in fluorescence intensity of a thallium-sensitive dye. B Time course of changes in fluorescence intensity reflecting the rate of thallium influx upon stimulation of cells with dopamine (1 $\mu \mathrm{M})$. C Concentration response relationship of GIRK-mediated thallium flux defined by quantifying changes in fluorescence slope (initial activation rate). D Quantification of the $\mathrm{EC}_{50}$ and maximum amplitude of thallium flux in D2R-expressing HEK293T cells with or without GPR139. E Cell surface abundance of HiBiT-D2RL measured in the absence and presence of GPR139. Data are mean \pm S.E.M. of 4-5 independent experiments. Data were analyzed by two-tailed Student's $t$ test, ${ }^{*} p<0.05,{ }^{* *} p<0.01$, n.s., $p>0.05$. F-J The effects of D2R blockade with haloperidol on locomotor activity $(\mathbf{E})$, head twitching $(\mathbf{F})$, pre-pulse inhibition $(\mathbf{G})$ and novel object recognition $(\mathbf{H}, \mathbf{I})$. In novel object recognition test, mice were presented first with the same objects and the following day one object was replaced with a new one. Haloperidol was administered immediately prior to testing in both sessions. Light gray and light red: interaction with the familiar object (gray square); dark gray and dark red: interaction with novel object (blue cylinder). Six mice of both sexes were evaluated per each group as indicated, ${ }^{* * * *} p<0.0001$; data sets (mean \pm SEM) were analyzed using unpaired two tailed Student $t$ test and paired two tailed Student's $t$ test for comparison within genotype. In panels $\mathbf{F}-\mathbf{J}$ male data are in squares, female - circles.

behavioral manifestations related to schizophrenia. Many of these observations form a foundation for the conclusion that the habenula plays an important role in schizophrenia development [50]. Thus, it is tempting to speculate that many behavioral deficits that we observe are related to deregulation of habenular function. However, the present study did not examine the circuits responsible for phenotypes associated with the loss of GPR139 whose expression is certainly not limited to habenula. Thus, the hypothesis regarding habenula involvement in driving the GPR139 phenotypes will need to be tested directly when region and celltype specific manipulation with GPR139 expression becomes possible. 
One of the key observations of our study is that a subset of neuropsychiatric behavioral abnormalities in GPR139 knockout mice could be pharmacologically reversed. This finding has several implications. First, it argues that several behavioral alterations in this mouse model are not a result of developmental adaptations as they could be normalized in adult subjects. Second, the compounds that were effective in correcting abnormal behaviors antagonize GPCRs, indicating that behavioral manifestations were likely caused by excessive neuromodulatory signaling. Third, the results implicate dopaminergic and opioidergic systems in the effects of GPR139 on behavior, in particular involving D2R and MOR receptors. Indeed, GPR139 has been shown to intersect with both MOR and D2R by virtue of co-expression in the same neuronal populations, effects on signaling and in the case of MOR, physical association $[26,28]$. In particular, GPR139 was found to employ several mechanisms to antagonize MOR signaling and its inhibitory effects on neuronal firing in habenula neurons, most prominently involving antagonism between $\mathrm{Gq} / 11$ activated by GPR139 and Gi/o activated by MOR at their common effectors [25]. Given similar Gi/o-coupling of D2R, we think similar effector antagonism mechanisms could explain inhibitory influence of GPR139 on D2R.

Conceptually, these observations suggest that behavioral anomalies seen upon loss of GPR139 could be triggered by excessive signaling through MOR and D2R augmenting processing of endogenous opioidergic and dopaminergic neuromodulation. This idea is supported by the observations that pharmacological activation of these systems by opioid drugs and psychostimulants produce very similar behavioral effects including hyperactivity, PPI deficits and cognitive impairments [57, 58]. Furthermore, dopaminergics, (e.g., amphetamines) are precipitating factors for psychosis and delusions in humans $[59,60]$ and are commonly used to model schizophrenia in rodents [38]. Finally, genetic augmentation of $D 2 R$ in the striatum also models many schizophrenia-like endophenotypes in mice [61]. Loss of GPR139 may distinctly augment both D2R and MOR systems in parallel, which could synergize to produce behavioral effects. Alternatively, augmentation of MOR and D2R may be interrelated, e.g., MOR activation is well known to augment dopaminergic signaling through "disinhibition" effect on circuits [62]. From that perspective, the contribution of augmented opioid signaling to schizophrenia-like phenotype of GPR139 knockouts seems intriguing for two reasons. First, activation of MOR negatively regulates the NMDA-type glutamate receptor [63-65], a target heavily implicated in schizophrenia, whose inhibition or hypofunction produces hallucinations and many cardinal features of the disease [66]. Second, suppression of opioid signaling has been a focus of many clinical trials for interventions in schizophrenia [67]. The mixed success of these trials may be related to the varying nature of the underlying molecular factors. Disruptions in negative regulators of MOR, such as GPR139, may lead to hyperfunction of opioidergic system making only some cases of schizophrenia responsive to opioid antagonists. While the links of GPR139 to schizophrenia in humans remain to be established, it might be interesting to consider it among schizophrenia risk factors and its responsiveness to opioid modulation. As a step in this direction, our behavioral data provide the first experimental evidence implicating the poorly understood orphan receptor GPR139 in fundamental neuropsychiatric processes with possible implications for understanding schizophrenia pathology.

\section{FUNDING AND DISCLOSURE}

This work was supported by the NIH grant DA048036 and MH105482 (KAM) and DA047771 (HMS). The funders had no influence over the content of this publication. KAM has filed a patent $62 / 746,343$ pertaining to the utility of pharmacological targeting of GPR139. KAM also provides consulting services regarding the utility of GPR139 as a drug target to EvoDenovo, Inc and owns shares of that company. The authors have no other competing financial interests in relation to the work described.

\section{ACKNOWLEDGEMENTS}

The authors thank Natalia Martemyanova for technical help with mouse husbandry and members of Martemyanov laboratory for helpful discussions. We also wish to thank Drs. Henry Dunn and Subhi Marwari for critical comments on the manuscript.

\section{AUTHOR CONTRIBUTIONS}

MD carried out all of the behavioral experiments, analyzed the data and participated in writing the manuscript. HMS performed signaling assays, analyzed the data and edited the manuscript. YC performed water maze experiments, analyzed the data, and edited the manuscript. KAM conceived the study, analyzed the data and wrote the manuscript.

\section{ADDITIONAL INFORMATION}

Publisher's note Springer Nature remains neutral with regard to jurisdictional claims in published maps and institutional affiliations.

\section{REFERENCES}

1. Marder E. Neuromodulation of neuronal circuits: back to the future. Neuron. 2012;76:1-11.

2. Bargmann $\mathrm{Cl}$, Marder E. From the connectome to brain function. Nat Methods. 2013;10:483-90.

3. Avery MC, Krichmar JL. Neuromodulatory systems and their interactions: a review of models, theories, and experiments. Front Neural Circuits. 2017;11:108.

4. Marinissen MJ, Gutkind JS. G-protein-coupled receptors and signaling networks: emerging paradigms. Trends Pharmacol Sci. 2001;22:368-76.

5. Lefkowitz RJ. Seven transmembrane receptors: something old, something new. Acta Physiol. 2007;190:9-19.

6. Sriram K, Insel PA. G protein-coupled receptors as targets for approved drugs: how many targets and how many drugs? Mol Pharmacol. 2018;93:251-58.

7. Hauser AS, Chavali S, Masuho I, Jahn LJ, Martemyanov KA, Gloriam DE, et al. Pharmacogenomics of GPCR drug targets. Cell. 2018;172:41-54.e19.

8. Catapano LA, Manji HK. G protein-coupled receptors in major psychiatric disorders. Biochim Biophys Acta. 2007;1768:976-93.

9. Tomita H, Ziegler ME, Kim HB, Evans SJ, Choudary PV, Li JZ, et al. G protein-linked signaling pathways in bipolar and major depressive disorders. Front Genet. 2013;4:297.

10. Civelli O. Orphan GPCRs and neuromodulation. Neuron. 2012;76:12-21.

11. Laschet $C$, Dupuis $N$, Hanson J. The $G$ protein-coupled receptors deorphanization landscape. Biochem Pharmacol. 2018;153:62-74.

12. Vedel L, Nohr AC, Gloriam DE, Brauner-Osborne H. Pharmacology and function of the orphan GPR139 G protein-coupled receptor. Basic Clin Pharmacol Toxicol. 2020;126(Suppl 6):35-46.

13. Gloriam DE, Schioth $H B$, Fredriksson R. Nine new human Rhodopsin family Gprotein coupled receptors: identification, sequence characterisation and evolutionary relationship. Biochim Biophys Acta. 2005;1722:235-46.

14. Foster SR, Hauser AS, Vedel L, Strachan RT, Huang XP, Gavin AC, et al. Discovery of human signaling systems: pairing peptides to $G$ protein-coupled receptors. Cell 2019;179:895-908.e21.

15. Nohr AC, Shehata MA, Hauser AS, Isberg V, Mokrosinski J, Andersen KB, et al. The orphan $G$ protein-coupled receptor GPR139 is activated by the peptides: adrenocorticotropic hormone (ACTH), alpha-, and beta-melanocyte stimulating hormone (alpha-MSH, and beta-MSH), and the conserved core motif HFRW. Neurochem Int 2017;102:105-13.

16. Liu C, Bonaventure $P$, Lee $G$, Nepomuceno D, Kuei $C$, Wu J, et al. GPR139, an orphan receptor highly enriched in the habenula and septum, is activated by the essential amino acids L-tryptophan and L-phenylalanine. Mol Pharmacol. 2015;88:911-25.

17. Hu LA, Tang PM, Eslahi NK, Zhou T, Barbosa J, Liu Q. Identification of surrogate agonists and antagonists for orphan G-protein-coupled receptor GPR139. J Biomol Screen. 2009;14:789-97.

18. Isberg V, Andersen KB, Bisig C, Dietz GP, Brauner-Osborne $H$, Gloriam DE. Computer-aided discovery of aromatic I-alpha-amino acids as agonists of the orphan G protein-coupled receptor GPR139. J Chem Inf Model. 2014;54:1553-7. 
19. Dvorak CA, Coate H, Nepomuceno D, Wennerholm M, Kuei C, Lord B, et al. Identification and SAR of glycine benzamides as potent agonists for the GPR139 receptor. ACS Med Chem Lett. 2015;6:1015-8.

20. Wang J, Zhu LY, Liu Q, Hentzer M, Smith GP, Wang MW. High-throughput screening of antagonists for the orphan G-protein coupled receptor GPR139. Acta Pharm Sin. 2015;36:874-8.

21. Shehata MA, Nohr AC, Lissa D, Bisig C, Isberg V, Andersen KB, et al. Novel agonist bioisosteres and common structure-activity relationships for the orphan $G$ protein-coupled receptor GPR139. Sci Rep. 2016;6:36681.

22. Nohr AC, Shehata MA, Palmer D, Pokhrel R, Vallianou M, Foster SR, et al. Identification of a novel scaffold for a small molecule GPR139 receptor agonist. Sci Rep. 2019;9:3802.

23. Shoblock JR, Welty N, Fraser I, Wyatt R, Lord B, Lovenberg T, et al. In vivo characterization of a selective, orally available, and brain penetrant small molecule GPR139 agonist. Front Pharmacol. 2019;10:273.

24. Nohr AC, Jespers W, Shehata MA, Floryan L, Isberg V, Andersen KB, et al. The GPR139 reference agonists $1 \mathrm{a}$ and $7 c$, and tryptophan and phenylalanine share a common binding site. Sci Rep. 2017;7:1128.

25. Stoveken HM, Zucca S, Masuho I, Grill B, Martemyanov KA. The orphan receptor GPR139 signals via Gq/11 to oppose opioid effects. J Biol Chem. 2020;295:10822-30.

26. Wang L, Lee G, Kuei C, Yao X, Harrington A, Bonaventure P, et al. GPR139 and dopamine D2 receptor co-express in the same cells of the brain and may functionally interact. Front Neurosci. 2019;13:281.

27. Matsuo A, Matsumoto S, Nagano M, Masumoto KH, Takasaki J, Matsumoto $M$ et al. Molecular cloning and characterization of a novel Gq-coupled orphan receptor GPRg1 exclusively expressed in the central nervous system. Biochem Biophys Res Commun. 2005;331:363-9.

28. Wang D, Stoveken HM, Zucca S, Dao M, Orlandi C, Song C, et al. Genetic behavioral screen identifies an orphan anti-opioid system. Science. 2019;365:1267-73.

29. Kononoff J, Kallupi M, Kimbrough A, Conlisk D, de Guglielmo G, George O Systemic and intra-habenular activation of the orphan $G$ protein-coupled receptor GPR139 decreases compulsive-like alcohol drinking and hyperalgesia in alcohol-dependent rats. eNeuro. 2018;5:ENEURO.0153-18.2018.

30. Curzon PR, NR; Browman, KE. Cued and contextual fear conditioning for Rodents. Boca Raton, FL: CRC Press/Taylor \& Francis; 2009.

31. Cao Y, Sarria I, Fehlhaber KE, Kamasawa N, Orlandi C, James KN, et al. Mechanism for selective synaptic wiring of rod photoreceptors into the retinal circuitry and its role in vision. Neuron. 2015;87:1248-60.

32. Fowler CD, Kenny PJ. Intravenous nicotine self-administration and cue-induced reinstatement in mice: effects of nicotine dose, rate of drug infusion and prior instrumental training. Neuropharmacology. 2011;61:687-98.

33. Sutton LP, Muntean BS, Ostrovskaya O, Zucca S, Dao M, Orlandi $C$, et al. NF1-cAMP signaling dissociates cell type-specific contributions of striatal medium spiny neurons to reward valuation and motor control. PLoS Biol. 2019;17:e3000477.

34. Clapcote SJ, Lipina TV, Millar JK, Mackie S, Christie S, Ogawa F, et al. Behavioral phenotypes of Disc1 missense mutations in mice. Neuron. 2007;54:387-402.

35. Hikida T, Jaaro-Peled H, Seshadri S, Oishi K, Hookway C, Kong S, et al. Dominantnegative DISC1 transgenic mice display schizophrenia-associated phenotypes detected by measures translatable to humans. Proc Natl Acad Sci USA. 2007; 104:14501-6.

36. Harrison PJ, Law AJ. Neuregulin 1 and schizophrenia: genetics, gene expression, and neurobiology. Biol Psychiatry. 2006;60:132-40.

37. Mei L, Xiong WC. Neuregulin 1 in neural development, synaptic plasticity and schizophrenia. Nat Rev Neurosci. 2008;9:437-52.

38. Jones CA, Watson DJ, Fone KC. Animal models of schizophrenia. Br J Pharmacol. 2011;164:1162-94.

39. Canal CE, Morgan D. Head-twitch response in rodents induced by the hallucinogen 2,5-dimethoxy-4-iodoamphetamine: a comprehensive history, a re-evaluation of mechanisms, and its utility as a model. Drug Test Anal. 2012;4:556-76.

40. Nabeshima T, Ishikawa K, Yamaguchi K, Furukawa H, Kameyama T. Phencyclidineinduced head-twitch response in rats treated chronically with methysergide. Eur J Pharmacol. 1987;133:319-28.

41. Corne SJ, Pickering RW. A possible correlation between drug-induced hallucinations in man and a behavioural response in mice. Psychopharmacologia. 1967; 11:65-78.

42. Jardri R, Pins D, Bubrovszky M, Lucas B, Lethuc V, Delmaire $C$, et al. Neural functional organization of hallucinations in schizophrenia: multisensory dissolution of pathological emergence in consciousness. Conscious Cogn. 2009;18:449-57.

43. Miller JN, Black DW. Schizoaffective disorder: a review. Ann Clin Psychiatry. 2019;31:47-53.
44. Castellani CA, Awamleh Z, Melka MG, O'Reilly RL, Singh SM. Copy number variation distribution in six monozygotic twin pairs discordant for schizophrenia. Twin Res Hum Genet. 2014;17:108-20.

45. Atienza TR H, Mulligan V, Cilia J, Monenschein H, Collia D, Ray J, et al. GPR139 an ophan GPCR affecting negative domains of schizophrenia. Schizophrenia Bull. 2018;44:S339.

46. Schiffer HA J, Reichard H, Mulligan V, Cilia J, Monenschein H, Collia D, et al. The selective GPR139 agonist TAK-041 reverses anhedonia and social interaction deficits in rodent models related to negative symptoms in schizophrenia. Schizophrenia Bull 2020;46:S106-S07.

47. Takeda. A randomized, double-blind, placebo controlled, two-period cross-over, proof of activity study to evaluate the effects of TAK-041 on motivational anhedonia as add-on to antipsychotics in subjects with stable schizophrenia. https://clinicaltrialsgov/ct2/show/NCT03319953. 2019; ClinicalTrials.gov Identifier: NCT03319953.

48. Susens U, Hermans-Borgmeyer I, Urny J, Schaller HC. Characterisation and differential expression of two very closely related G-protein-coupled receptors, GPR139 and GPR142, in mouse tissue and during mouse development. Neuropharmacology. 2006;50:512-20.

49. McLaughlin I, Dani JA, De, Biasi M. The medial habenula and interpeduncular nucleus circuitry is critical in addiction, anxiety, and mood regulation. J Neurochem. 2017;142:130-43.

50. Fakhoury M. The habenula in psychiatric disorders: more than three decades of translational investigation. Neurosci Biobehav Rev. 2017;83:721-35.

51. Sandyk R. Pineal and habenula calcification in schizophrenia. Int J Neurosci. 1992;67:19-30.

52. Shepard PD, Holcomb HH, Gold JM. Schizophrenia in translation: the presence of absence: habenular regulation of dopamine neurons and the encoding of negative outcomes. Schizophr Bull. 2006;32:417-21.

53. van Kerkhof LW, Damsteegt R, Trezza V, Voorn P, Vanderschuren LJ. Functional integrity of the habenula is necessary for social play behaviour in rats. Eur $J$ Neurosci. 2013;38:3465-75.

54. Madularu D, Kulkarni P, Yee JR, Kenkel WM, Shams WM, Ferris CF, et al. High estrogen and chronic haloperidol lead to greater amphetamine-induced BOLD activation in awake, amphetamine-sensitized female rats. Horm Behav. 2016;82:56-63.

55. Heldt SA, Ressler KJ. Lesions of the habenula produce stress- and dopaminedependent alterations in prepulse inhibition and locomotion. Brain Res. 2006 1073-1074:229-39.

56. Stopper CM, Tse MTL, Montes DR, Wiedman CR, Floresco SB. Overriding phasic dopamine signals redirects action selection during risk/reward decision making Neuron. 2014;84:177-89.

57. Gainetdinov RR. Dopamine transporter mutant mice in experimental neuropharmacology. Naunyn-Schmiedeberg's Arch Pharmacol. 2008;377:301-13.

58. Charbogne P, Kieffer BL, Befort K. 15 years of genetic approaches in vivo for addiction research: Opioid receptor and peptide gene knockout in mouse models of drug abuse. Neuropharmacology. 2014;76:204-17.

59. Maremmani AG, Rovai L, Rugani F, Bacciardi S, Dell'Osso L, Maremmani I. Substance abuse and psychosis. The strange case of opioids. Eur Rev Med Pharm Sci. 2014;18:287-302.

60. Mullen JM, Richards JR, Crawford AT. Amphetamine related psychiatric disorders Treasure Island (FL): StatPearls;2020.

61. Simpson EH, Kellendonk C. Insights about striatal circuit function and schizophrenia from a mouse model of dopamine D2 receptor upregulation. Biol Psychiatry. 2017;81:21-30.

62. Nestler EJ. Is there a common molecular pathway for addiction? Nat Neurosci. 2005;8:1445-9.

63. Rusin KI, Randic M. Modulation of NMDA-induced currents by mu-opioid receptor agonist DAGO in acutely isolated rat spinal dorsal horn neurons. Neurosci Lett. 1991;124:208-12.

64. Martin G, Nie Z, Siggins GR. mu-Opioid receptors modulate NMDA receptormediated responses in nucleus accumbens neurons. J Neurosci. 1997;17:11-22.

65. Xie CW, Lewis DV. Involvement of CAMP-dependent protein kinase in muopioid modulation of NMDA-mediated synaptic currents. J Neurophysiol. 1997; 78:759-66.

66. Lee G, Zhou Y. NMDAR hypofunction animal models of schizophrenia. Front Mol Neurosci. 2019;12:185.

67. Clark SD, Van Snellenberg JX, Lawson JM, Abi-Dargham A. Opioid antagonists are associated with a reduction in the symptoms of schizophrenia: a meta-analysis of controlled trials. Neuropsychopharmacology. 2020;45:1860-9. 